\title{
La Escuela Nacional de Medicina del Trabajo como centro nacional de referencia en la formación de medicina y enfermería del trabajo
}

\section{National School of Occupational Medicine as reference in the formation of me- dicine and nursing labour}

\author{
Jerónimo Maqueda Blasco ', Rosa Ana Cortés Barragán 2 , María Fe Gamo González ${ }^{3}$ \\ 1. Director de la Escuela Nacional de Medicina del Trabajo (ENMT) del Instituto de Salud Carlos III (ISCIIII. \\ Madrid. España. \\ 2. Jefa de Estudios de Post grado en Salud Laboral. ENMT-ISCIII. Madrid. España. \\ 3. Coordinadora Docente. ENMT-ISCIII. Madrid. España.
}

Recibido: 20-06-12

Aceptado: 27-06-12

\section{Correspondencia:}

Jerónimo Maqueda Blasco

Escuela Nacional de Medicina del Trabajo

Instituto de Salud Carlos III

Pabellón 8, Ciudad Universitaria

28040 Madrid. España.

E-mail: direccion.enmt@isciii.es

Hablar de los últimos seis años de la Escuela Nacional de Medicina de Trabajo (ENMT), supone hablar de un periodo de trabajo para afrontar una adaptación de la actividad y carta de servicios de la Escuela a las nuevas necesidades en el ejercicio de la de Medicina y Enfermería del Trabajo.

El conjunto de esfuerzos desarrollados por el equipo de la ENMT ha estado dirigido a llevar la Escuela al centro y al puesto de trabajo del profesional de la Medicina y Enfermería, invirtiendo las anteriores coordenadas bajo las cuales era el profesional el que se debía acercar a la Escuela.

Los pilares en los que se ha basado la política de desarrollo de la Escuela Nacional de Medicina del Trabajo han sido: la innovación tecnológica, el fortalecimiento de la oferta docente y las alianzas con los diferentes actores comprometidos con el progreso en salud de la sociedad del trabajo de nuestro país

El desarrollo de la ENMT se ha realizado en el marco de la Sociedad de la Información, siendo la utilización de las TICs el mejor vehículo para llevar el conocimiento científico y la formación al puesto de trabajo del profesional sanitario.

Este recorrido se inició con una profunda modificación en la gestión editorial de la Revista Medicina y Seguridad del Trabajo, trasladándola de un escenario de edición escrita bajo subscripción a un nuevo escenario de edición electrónica (incluido el formato de e-book) y acceso abierto (Open Access), ambas medidas acompañadas de la internacionalización de su comité editorial. Actualmente a la Revista de Medicina y Seguridad del Trabajo es una de las publicaciones periódicas editadas en español con mayor nivel de indexación en las bases de datos bibliográficas y repositorios nacionales e internacionales de la producción científica en el marco de la Salud Laboral y la Medicina del Trabajo.

El proyecto de InfoSaludLaboral (http://new.paho.org/isl), portal de acceso a fuentes de información de interés en Salud Laboral, permite al profesional de medicina o enfermería enlazar desde el mismo portal Web a repertorios de revistas, bases de datos bibliográficas, observatorios y redes de información y URL de instituciones nacionales e internacionales relacionadas con la Seguridad y Salud en el Trabajo. 
La constitución de la Comisión Docente, como órgano académico colegiado de asesoramiento, supone una de las aportaciones más importantes en la modernización estructural de la ENMT.

El fortalecimiento de la actividad docente ha sido fruto de tres líneas de trabajo: - la consolidación del Curso Superior de Medicina del Trabajo, la alineación de la oferta de formación continua con la Ley de Ordenación de las Profesiones Sanitarias y el desarrollo de programas de formación de posgrado dentro del Espacio Europeo de Educación Superior.

Estas tres líneas de trabajo han permitido la renovación de proyectos de formación existentes, como por ejemplo el Diploma de Valoración Médica de la Incapacidad Laboral, que mediante convenio con la Universidad de Alcalá de Henares y la participación y colaboración del Instituto Nacional de la Seguridad Social (INSS) y del Instituto de Mayores y Servicios Sociales (IMSERSO) de España, aparecerá en el próximo año académico como Master en Valoración Médica de la Incapacidad laboral y Dependencia, abordando nuevas áreas de evaluación médica necesarias en el actual campo socio-sanitario. Dentro de esta oferta docente compartida con la Universidad se inicia también en el próximo año académico el Master Oficial de Prevención de Riesgos Laboral (especialidad en psico-sociología) en colaboración con la Facultad de Psicología de la Universidad Complutense de Madrid. El Master en Valoración Médica de la Incapacidad con la Universidad Miguel Hernández y el Título de Experto Universitario en Enfermedades Profesionales con la Universidad de Islas Baleares, cierran la línea de la oferta docente de la ENMT vinculada a la Universidad.

La intensificación de la formación continua en el marco dela Ley 44/23 de Ordenación de las Profesiones Sanitarias (LOPS) como medio de contribución a la sostenibilidad de las competencias y desarrollo de la carrera profesional, ha ocupado un espacio de atención preferente por parte del equipo de la Jefatura de Estudios de la ENMT.

Esta línea ha supuesto un importante esfuerzo en la sistematización y apuesta por la calidad, ya que todos los cursos de formación continua de la ENMT pasan un proceso de acreditación ante la Comisión de Formación Continua de las profesiones sanitarias.

El paso más importante en nuestros esfuerzos por llegar al profesional en ejercicio se ha dado con la creación en 2010 del Campus e-ENMTde Formación en Medicina y Enfermería del Trabajo (http:// campus.enmt.otp.es). Este proyecto de "e-aprendizaje" tiene como objetivo facilitar el acceso a una formación de calidad a todos los profesionales sanitarios de prevención de riesgos laborales, con independencia del ámbito geográfico o entorno profesional en el que desarrolla su ejercicio.

El Campus e-ENMT integra en la actualidad siete cursos que suman un total de 315 horas docentes y30 créditos de formación continuada (CFC). Intervienen un total de 38 tutores y ha reunido en 2011 a un total de 333 alumnos.

De forma añadida la actividad de formación continua de carácter presencial se ha intensificado pasando de 3 cursos en 2007 a 17 en 2011.

La intensificación de la actividad formativa se refleja en los indicadores de horas lectivas que han pasado de 1.720 en 2007 a 2.707 en 2011 y en el número de profesionales que han recibido formación en la ENMT que han pasado de 169 en 2007 a 1.198 en 2011

La formación del Médico especialista en Medicina del Trabajo constituye uno de los proyectos formativos más consolidados de la ENMT.

La formación de Médicos Internos Residentes (MIR) dentro de la ENMT tiene dos áreas de desarrollo: El Curso Superior de Medicina del Trabajo (CUSMET) y el Programa de Investigación asociado al Programa MIR.

La solidez de este proyecto viene dada por el hecho de haberse construido progresivamente con la revisión y aportaciones de los Jefes de Estudios de las 11 Unidades Docentes de Medicina del Trabajo de las que la ENMT actúa como Centro Formativo de Referencia; este proceso lo convierte en el proyecto de formación MIR más sólido de nuestro país.

Los indicadores de satisfacción del MIR, tanto con los contenidos como con el conjunto de expertos docentes que intervienen, son una evidencia de la importancia de este tipo de actividad formativa presencial dentro del programa oficial de la especialidad, reflejados en la evaluación de la actividad y en la opinión de los propios residentes. 
Dentro de la formación especializada la ENMT pone a disposición del MIR un total de 115 expertos, 29 tutores y 82 evaluadores en los programas de investigación, manteniendo una 159 residentes en diferentes años de especialización. Con una producción desde el inicio del nuevo modelo de residencia de: 157 protocolos de actuación preventiva, 83 trabajos de revisión y 108 proyectos de investigación.

Estos datos ponen de manifiesto la gran fortaleza de la "Comunidad Académica" de la ENMT, y que está en el camino adecuado para cumplir su misión como Centro Nacional de Referencia en materia formativa en Medicina y Enfermería del Trabajo.

El compromiso de la ENMT con el profesional sanitario de prevención de riesgos laborales incluye el apoyo al profesional en ejercicio profesional mediante una línea de mejora de la práctica clínica a través del desarrollo de estudios, proyectos de investigación o guías de práctica clínica, desarrolladas en colaboración con Sociedades científicas y Profesionales, como la Asociación Española de Especialistas de Medicina del Trabajo (AEEMT), la Asociación de Medicina del Trabajo del Ámbito Sanitario (ANMTAS), la Asociación Madrileña de Medicina de Familia y Comunitaria (SoMaMFyC) y colaboración con Instituciones del Estado y de la Seguridad Social (MUTUAS, INSS, INSHT, INVASSAT, etc.) e Interlocutores sociales (UGT, CCOO, ISTAS, FECOMA, MCA-UGT, CONFEMAERA, etc.).

En la actualidad, la ENMT afronta nuevos compromisos tanto en la mejora continua de calidad, profundizar en los desarrollos tecnológicos adaptando sistemas de telemedicina y e-Salud a sus diferentes servicios. La formación en la Especialidad de Enfermería del Trabajo y la Internacionalización de su oferta formativa iniciada ya en colaboración con la Organización Panamericana de la Salud, Oficina regional para las Américas de la Organización Mundial de la Salud (OPS/OMS), ampliándola a otras instituciones internacionales como la Organización Internacional del Trabajo (OIT) o la Asociación Internacional de la Seguridad Social (AISS).

Pero sin lugar a dudas, la tarea principal que abordamos continúa siendo el intento continuado y sostenido de llegar a todos los profesionales de la Medicina y Enfermería del Trabajo y contribuir a facilitar y mejorar su ejercicio profesional a través del conocimiento. 